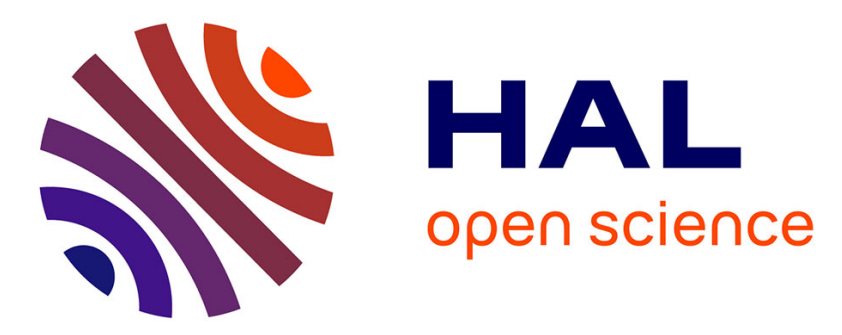

\title{
Parameter estimation of pharmacokinetics models in the presence of timing noise
}

Thierry Bastogne, Sophie Mézières-Wantz, Nacim Ramdani, Pierre Vallois, Muriel Barberi-Heyob

\section{- To cite this version:}

Thierry Bastogne, Sophie Mézières-Wantz, Nacim Ramdani, Pierre Vallois, Muriel Barberi-Heyob. Parameter estimation of pharmacokinetics models in the presence of timing noise. European Control Conference, ECC'07, Jul 2007, Kos, Greece. pp.CDROM. hal-00141540

\author{
HAL Id: hal-00141540 \\ https://hal.science/hal-00141540
}

Submitted on 13 Apr 2007

HAL is a multi-disciplinary open access archive for the deposit and dissemination of scientific research documents, whether they are published or not. The documents may come from teaching and research institutions in France or abroad, or from public or private research centers.
L'archive ouverte pluridisciplinaire HAL, est destinée au dépôt et à la diffusion de documents scientifiques de niveau recherche, publiés ou non, émanant des établissements d'enseignement et de recherche français ou étrangers, des laboratoires publics ou privés. 


\title{
Parameter Estimation of Pharmacokinetics Models in the presence of Timing Noise
}

\author{
Thierry Bastogne, Sophie Mézières-Wantz, Nacim Ramdani, Pierre Vallois and Muriel Barberi-Heyob
}

\begin{abstract}
The problem addressed in this paper deals with the parameter estimation of in vitro uptake kinetics of drugs into living cells in presence of timing noise. Effects of the timing noise on the bias and variance of the output error are explicitly determined. A bounded-error parameter estimation approach is proposed as a suited solution to handle this problem. Application results are presented and emphasize its effectiveness in such an experimental framework.
\end{abstract}

Keywords: parameter estimation, pharmacokinetics models, timing errors, bounded errors, biological systems.

\section{INTRODUCTION}

Pharmacokinetics is the study of the bodily absorption, distribution, metabolism and excretion of drugs by bodies. In chemical kinetics, reactions are generally described by differential equations which link the reaction rate with concentrations or pressures of reactants. In molecular cell biology, because of the complexity of systems, the nature of some reactions is still unclear. This paper is focused on the intracellular uptake kinetics of a photosensitizing drug (PS), i.e. the rate of photosensitizing molecules incorporated and accumulated by living cancer cells according to incubation terms [1]. The delivery control of the photosensitizing agent into the cancer cells is one the major factor on the therapeutic efficiency of the photodynamic therapy (PDT) [2]. Most of the PS uptake kinetics models are non-parametric, the temporal evolution of the PS intracellular concentration is described by step responses. The purpose of this study is the estimation of kinetics model parameters from data collected during in vitro kinetics experiments. These parameters are crucial information to both improve the modalities of the drug delivery process in photodynamic therapy and discriminate the uptake characteristics of different photosensitizers. Few papers have been reported for the application of system identification techniques to pharmacokinetics modeling problems [3]-[5].

T. Bastogne is with the Centre de Recherche en Automatique de Nancy (CRAN), Nancy-Université, CNRS, BP 239, F-54506 Vandœuvre-lès-Nancy Cedex, France thierry.bastognedcran.uhp-nancy.fr

$\mathrm{S}$. Mézières-Wantz and P. Vallois are with the Institut de Mathématiques Elie Cartan, Nancy-Université, CNRS, BP 239, F-54506 Vandœuvrelès-Nancy Cedex, France sophie.wantzeiecn.u-nancy.fr, pierre.valloisdiecn.u-nancy.fr

N. Ramdani was with the CERTES EA 3481 Université Paris 12 Val de Marne. He is now with the Laboratoire d'Informatique, de Robotique et de Microélectronique de Montpellier (LIRMM) UMR CNRS 5506 Université de Montpellier 2, 161 rue Ada, 34392 Montpellier Cedex 5 - France. nacim.ramdani@lirmm. fr

M. Barberi-Heyob is with the Centre de Recherche en Automatique de Nancy (CRAN), Nancy-Université, CNRS, Centre Alexis Vautrin, 54511 Vandœuvre-lès-Nancy Cedex, France m.barberi@nancy.fnclcc.fr
Unfortunately, these approaches cannot be applied to the PS uptake kinetics. The intracellular PS concentration $\left(\left[P_{i}\right]\right)$ is measured by a spectrofluorimeter but the latter induces a photobleaching process of the PS. The term photobleaching refers to the process by which the chromophoric structure of the PS is degraded by absorbed light energy [6]. As PS can be photobleached after light exposure, repeated experimentations for the same biological sample are not conceivable. In other terms, one biological sample with PS cannot be used for consecutive measurements of $\left[P_{i}\right]$. Collecting $N_{t}$ data points of the kinetics then requires to repeat $N_{t}$ times the same experiment $\left(N_{t}\right.$ biological samples) with identical initial conditions. To avoid the time consuming and the too high cost of such an experiment set up, $N_{t}$ is generally kept small, i.e. $N_{t} \leq 10$. This limitation on $N_{t}$ is the first problem to overcome for estimating kinetics parameters. The second difficulty is the low signal-to-noise ratio. The latter is due to a great measurement variability when working on living cells which are very sensitive to external disturbances. Thirdly, the choice of the stimulus signal is restricted to step signals which correspond to the amount of PS injected into the culture medium wells at time $t=0$. A last issue is a timing offset error in the measurement samples. This timing error is bounded and can reach until $\pm 15 \mathrm{mn}$ for a measurement time sequence $\left\{t_{j}\right\}=\{1,2,4,6,8,14,18,24 h\}$. This error is not negligible and has rarely been taken into account in parameter estimation problems [7], particularly in this restricted experimental framework.

The problem addressed in this paper deals with the parameter estimation of pharmacokinetic models in presence of timing errors. Parameter estimation algorithms in system identification methods are often based on the minimization of a quadratic function of the output error, i.e. the difference between the system and the model outputs. The sensitivity of the output error to timing noise comparatively to input and output noise is unknown. The contributions of this paper are twofold:

- the stochastic effects of the timing noise on the output error are explicitly determined. They are compared with the ones of input and output noise. These results are obtained by assuming that there is no modeling error between the model and the biological system;

- a solution suited to this system identification problem is proposed and applied to in vitro data sets in a second part of this paper in order to assess its effectiveness in practice. The identification problem is addressed in the bounded-error context and is solved with a set pro- 


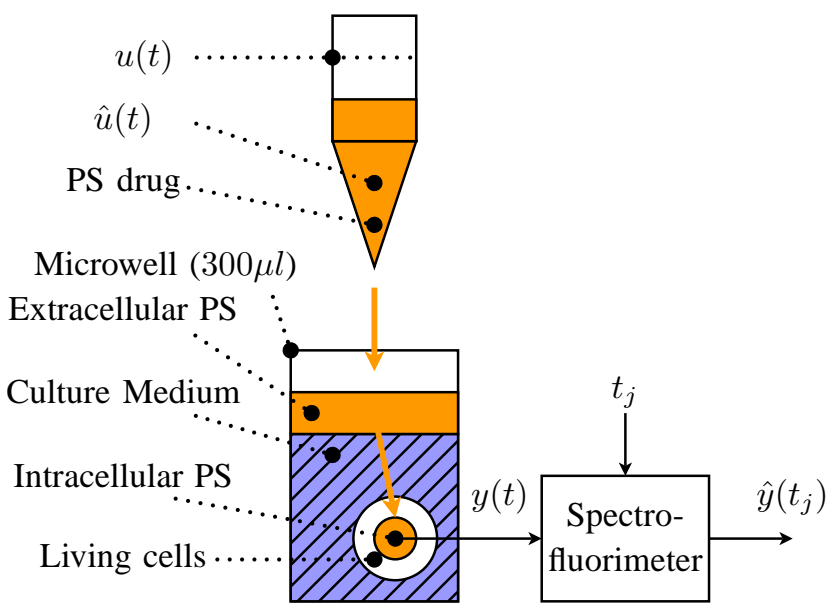

Fig. 1. in vitro experimental set up

jection algorithm based on interval analysis introduced in [8].

\section{EXPERIMENTAL SET UP}

TABLE I

MAIN NOTATIONS

\begin{tabular}{ll}
\hline Symb. & Description \\
\hline$t$ & time variable \\
$t_{j}$ & theoretical time instant \\
& associated with the $j^{t h}$ measurement sample \\
$\hat{t}_{j}$ & real time instant \\
& associated with the $j^{t h}$ measurement sample \\
$u(t)$ & noise-free input signal \\
& (stipulated by the experimenter) \\
$\hat{u}(t)$ & real input signal \\
$y(t)$ & system output variable \\
$\hat{y}(t)$ & (unknown by the experimenter) \\
$y_{\mathcal{M}}(t)$ & measured output variable \\
$n_{u}$ & model output variable \\
$n_{y}$ & input noise \\
$n_{t}$ & output noise \\
$e_{y}(t)$ & timing noise \\
$\mathcal{S}$ & output error variable \\
$\mathcal{M}(p)$ & biological system \\
$p$ & parametric model \\
$N_{t}=\operatorname{card}\left(\left\{t_{j}\right\}\right)$ & vector of parameters \\
$N_{r}$ & number of data points \\
$x^{\prime}$ & number of repeated experiments \\
$\mathcal{N}(\mu, \sigma)$ & at each time instant \\
$\mathcal{U}(a, b)$ & transposition of $x$ \\
$\mathcal{E}\{\cdot\}$ & gaussian distribution with mean $\mu$ and \\
\hline & standard deviation $\sigma$ \\
& uniform distribution \\
& on the interval $[a ; b]$ \\
& mathematical expectation operator \\
\hline
\end{tabular}

Fig.1 depicts the basic material used in in vitro experiments for studying the uptake kinetics of a photosensitizing drug into living cells. Cells are seeded in $250 \mu \mathrm{l}$ culture wells and are exposed at time $t_{0}=0$ to a photosensitizing drug $D$. Let us consider the uptake phenomenon as a dynamic system. Its input variable $u(t)$ is a step signal which corresponds to the amount of drug injected into the well at time $t=0$. Its output variable $y(t)$ is the amount of drug absorbed by the cells. $\hat{y}(t)$ is the measurement of $y(t)$ given by

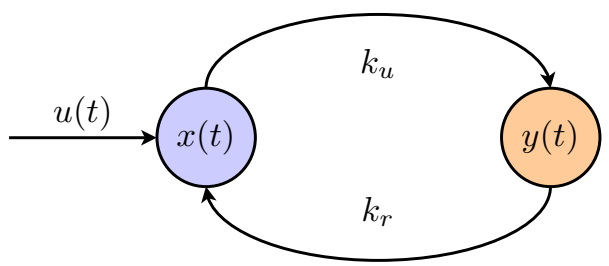

Fig. 2. in vitro compartmental model

a spectrofluorimeter at times $\left\{t_{j}\right\}$ with $j \in\left\{1, \cdots, N_{t}\right\}$. However, the spectrofluorimeter affects the biological state of the photosensitizing drug through a photobleaching process. Each culture well then becomes unusable after measurement. Consequently, to measure the intracellular PS concentration at $N_{t}$ different time instants, it is necessary to repeat the same experiment in $N_{t}$ different culture wells. Moreover, $N_{r}$ identical wells are handled by the experimenter at each time instant $t_{j}$ to $a$ posteriori estimate the repeatability of the measurements. Globally, $N_{r} \times N_{t}$ wells are handled during the whole experiment. In practice, such an experiment is also repeated for other PS and different concentrations of protein in the medium. Consequently, the total number of wells to handle can be much larger than $N_{r} \times N_{t}$. All the wells are prepared in the same initial conditions.

\section{III. in vitro PS UPTAKE MODELING}

The in vitro uptake of the PS agent into cancer cells can be described by a compartmental modeling approach. In this paper, a linear two compartments model presented in Fig.2, is used. The two compartments are associated with the extracellular and intracellular volumes respectively. $x(t)$ denotes the amount of extracellular PS. Parameters $k_{u}$ and $k_{r}$ are the uptake and release rates respectively. Differential equations of this compartmental model are defined as follows

$$
\begin{aligned}
& \frac{d x}{d t}=k_{r} y(t)-k_{u} x(t)+\frac{d u}{d t} \\
& \frac{d y}{d t}=k_{u} x(t)-k_{r} y(t),
\end{aligned}
$$

with $x(0)=y(0)=0$. After substitution of $x(t)$ from (1) in (2), it comes that

$$
\begin{aligned}
\frac{1}{k_{u}+k_{r}} \frac{d y}{d t}+y(t) & =\frac{k_{u}}{k_{u}+k_{r}} u(t), \quad \text { or } \\
T \frac{d y}{d t}+y(t) & =k u(t),
\end{aligned}
$$

where $T=1 /\left(k_{u}+k_{r}\right)$ and $k=k_{u} /\left(k_{u}+k_{r}\right)$ are the time constant and the static gain of the PS uptake model described by a linear first-order differential equation. In [9], it is shown that a first-order transfer function is indeed a parsimonious model structure for describing the uptake kinetics of the chlorin e6 photosensitizing drug into HT29-A4 cancer cells (human colon cancer cell line).

\section{MODEL AND ERRORS DESCRIPTIONS}

The determination of a parametric model describing the uptake kinetics of a photosensitizing drug into living cells 


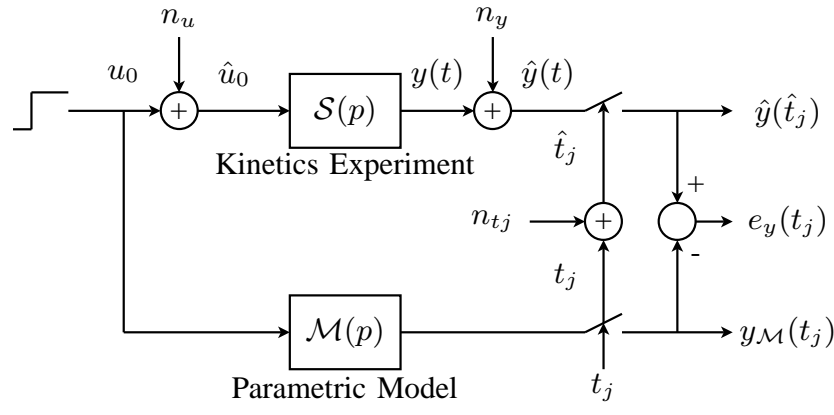

Fig. 3. Description of uncertainties

by extracting information from observations of $u$ and $y$ is a system identification problem [10]. At this point and thereafter, it is assumed that the system and the model are identical $\mathcal{M}(p) \equiv \mathcal{S}(p)$. However, as depicted in Fig. 3, three kinds of uncertainties are examined and are represented by output, input and timing noise (errors). Output and intput noise $\left(n_{y}\right.$ and $\left.n_{u}\right)$ are described by stationary stochastic processes added to the output and input signals. The timing noise $\left(n_{t}\right)$ is a sequence of random variables added to the timing sequence $\left\{t_{j}\right\}$ controlling the sampling process of the output signal. $\hat{t}_{j}$ is the real time instant at which the output variable $y$ is measured while $t_{j}$ represents the theoretical measurement time instant noted by the experimenter. $e_{y}\left(t_{j}\right)$ denotes the output error between the system and the model outputs $\left(\hat{y}\left(\hat{t}_{j}\right)\right.$ and $y_{\mathcal{M}}\left(t_{j}\right)$ respectively). Table I sums up the main notations used in the sequel.

\section{A. Model structure}

For the sake of simplicity, it is assumed in the sequel that $\mathcal{M}(p)$ and $\mathcal{S}(p)$ both rely on a first-order transfer function, inspired from (4),

$$
\begin{array}{ll}
\mathcal{S}(p): & T \cdot \frac{d y}{d t}+y(t)=k \cdot \hat{u}(t) \\
\mathcal{M}(p): & T \cdot \frac{d y_{\mathcal{M}}}{d t}+y_{\mathcal{M}}(t)=k \cdot u(t),
\end{array}
$$

with $y(0)=y_{\mathcal{M}}(0)=0 . p=(T, k)$ is the parameter vector where $T$ and $k$ denote the time constant and the static gain respectively. From a biological point of view, $T$ and $k$ inform the biologist about the uptake rate and yield respectvely. $u(t)$ is a step signal of magnitude $u_{0}$ defined in (10). As a result, the intracellular concentration of the photosensitizing drug $y$ follows a mono-exponential kinetics defined by

$$
y(t)=k \cdot \hat{u}_{0} \cdot\left(1-e^{-t / T}\right) .
$$

\section{B. Output noise}

Conjugated effects of measurement noise and disturbances are usually described by a stochastic variable $n_{y}$ added to $y$ such that

$$
\hat{y}\left(t_{j}\right)=y\left(t_{j}\right)+n_{y}\left(t_{j}\right),
$$

where $y$ and $\hat{y}$ denote the real biological response and its measurement respectively.

\section{Input noise}

$u$ is a step signal defined by

$$
u(t)= \begin{cases}0 & t<0 \\ u_{0} & t \geq 0\end{cases}
$$

The step magnitude $\left(u_{0}\right)$ represents the amount of the injected drug. The duration of injection is not significant compared to the duration of the experiment. The drug administration is usually carried out by multichannel micropipettes. For technical reasons, the real filling levels of drug in the cones are not identical and do not match with the dose stipulated by the experimenter. This error is represented by an input noise $n_{u}$ added to $u$ such that

$$
\hat{u}(t)= \begin{cases}0 & t<0 \\ \hat{u}_{0}=u_{0}+n_{u} & t \geq 0\end{cases}
$$

where $u_{0}$ and $\hat{u}_{0}$ denote the prescribed dose and the effectively administrated dose respectively.

\section{Timing noise}

Each experimentation is carried out in a sterile framework in which each well is carefully handled by the experimenter. For each time instant $t_{j}$ of the experimental set up, the living cells have to be washed, removed, lysed and diluted in ethanol by the experimenter. Such operations can take about $10 m n$ during which each well, among the $N_{r}$ ones, is individually handled. But the longest stage is the first one, i.e. the stage where the PS is administrated into all the wells; such an operation can take $20 \mathrm{mn}$. In practice, only the time instants $t_{j}^{-}$and $t_{j}^{+}$corresponding to the beginning and the end of the experiment are noted by the experimenter. The nominal measurement time instant $t_{j}$ noted by the experimenter in his table is an average time instant defined by $t_{j}=\left(t_{j}^{+}+t_{j}^{-}\right) / 2$. The real time instant $\hat{t}_{j}$ at which the uptake kinetics is stopped and measured, is unknown. This lack of precision in the timing of experiments is described by a timing noise $n_{t}$.

\section{Stochastic MODELING}

In this section it is assumed that $\left\{n_{y}\left(t_{j}\right)\right\}$ is an independent and identically distributed sequence of Gaussian variables

$$
n_{y}\left(t_{j}\right)=\sigma_{y} \cdot G_{y}^{j},
$$

where $\sigma_{y}$ denotes the standard deviation of $n_{y}$ and $G_{y}^{j} \approx$ $\mathcal{N}(0,1) \cdot n_{u}$ is supposed to be a Gaussian variable defined by

$$
n_{u}=\sigma_{u} \cdot G_{u},
$$

where $\sigma_{u}$ denotes the standard deviation of $n_{u}$ and $G_{u} \approx$ $\mathcal{N}(0,1)$. The timing noise sequence $\left\{n_{t j}\right\}$ is supposed to be independent and identically distributed sequence of uniform variables such that

$$
\begin{aligned}
n_{t j} & \approx \mathcal{U}\left(t_{j}^{-}, t_{j}^{+}\right) \\
& \approx-\frac{\tau_{j}}{2}+\tau_{j} \cdot U_{t}^{j},
\end{aligned}
$$


with $\tau_{j}=t_{j}^{+}-t_{j}^{-}$and $U_{t}^{j} \approx \mathcal{U}(0,1) . \tau_{j}$ denotes the width of the timing uncertainty interval for the time instant $t_{j}$. $\left\{n_{y}\left(t_{j}\right)\right\}, n_{u}$ and $\left\{n_{t j}\right\}$ are supposed to be independent. Given the previous assumptions about the input, output and timing noise, the expression of $e_{y}\left(t_{j}\right)$ becomes

$$
\begin{aligned}
e_{y}\left(t_{j}\right) & =\hat{y}\left(\hat{t}_{j}\right)-y_{\mathcal{M}}\left(t_{j}\right) \\
& =k \cdot\left(u_{0}+\sigma_{u} G_{u}\right) \cdot\left(1-e^{-\frac{1}{T}\left(t_{j}-\frac{\tau_{j}}{2}+\tau_{j} U_{t}^{j}\right)}\right)+\sigma_{y} G_{y}^{j} \\
& -k \cdot u_{0} \cdot\left(1-e^{-\frac{t_{j}}{T}}\right),
\end{aligned}
$$

where $k, T$ are given.

The mathematical expectation of $e_{y}\left(t_{j}\right)$ is defined in Proposition 5.1, its demonstration is developed in appendix II.

Proposition 5.1:

$$
\mathcal{E}\left\{e_{y}\left(t_{j}\right)\right\}=k \cdot u_{0} \cdot e^{\frac{-t_{j}}{T}}\left(1-\sinh c\left(\frac{\tau_{j}}{2 T}\right)\right),
$$

with $\sinh c(x)=\sinh (x) / x$ denotes the hyperbolic sinus cardinal function of $x$.

Since $x \rightarrow \operatorname{sinhc}(x)$ is increasing on $\mathbb{R}^{+}$, equation (17) shows that $\mathcal{E}\left\{e_{y}\left(t_{j}\right)\right\}<0$. This systematic bias is only due to the timing noise. The absolute value of the mean output error increases with respect to $\tau$ and is null only for $\tau=0$.

The variance of $e_{y}\left(t_{j}\right)$ is given in Proposition 5.2, its demonstration is developed in appendix III.

Proposition 5.2:

$\operatorname{Var}\left\{e_{y}\left(t_{j}\right)\right\}=\sigma_{y}^{2}+k^{2} \sigma_{u}^{2}+k^{2} e^{\frac{-t_{j}}{T}} \cdot \sinh c\left(\frac{\tau_{j}}{2 T}\right)(A+B)$,

with: $A=e^{\frac{-t_{j}}{T}}\left(\cosh \left(\frac{\tau_{j}}{2 T}\right)-\sinh c\left(\frac{\tau_{j}}{2 T}\right)\right) u_{0}^{2}$ and $B=$ $\left(e^{\frac{-t_{j}}{T}} \cosh \left(\frac{\tau_{j}}{2 T}\right)-2\right) \sigma_{u}^{2}$.

To take into account both the bias and the variance of $e_{y}\left(t_{j}\right)$, its mean square error defined by $\varepsilon\left(t_{j}\right)=$ $\mathcal{E}^{2}\left\{e_{y}\left(t_{j}\right)\right\}+\operatorname{Var}\left\{e_{y}\left(t_{j}\right)\right\}$ is examined thereafter. Three specific values of $\varepsilon\left(t_{j}\right)$, noted $\varepsilon_{n y}\left(t_{j}\right), \varepsilon_{n u}\left(t_{j}\right)$ and $\varepsilon_{n t}\left(t_{j}\right)$, are determined to emphasize the contribution of each kind of noise.

- $\sigma_{u}=0, \tau_{j}=0$ :

$$
\varepsilon_{n y}\left(t_{j}\right)=\sigma_{y}^{2}
$$

- $\sigma_{y}=0, \tau_{j}=0$ :

$$
\varepsilon_{n u}\left(t_{j}\right)=k^{2} \sigma_{u}^{2}\left(e^{\frac{-t_{j}}{T}}-1\right)^{2},
$$

- $\sigma_{y}=0, \sigma_{u}=0$ :

$$
\begin{aligned}
\varepsilon_{n t}\left(t_{j}\right) & =k^{2} u_{0}^{2} e^{\frac{-2 t_{j}}{T}}\left(1-2 \sinh c\left(\frac{\tau_{j}}{2 T}\right)\right. \\
& \left.+\sinh c\left(\frac{\tau_{j}}{2 T}\right) \cosh \left(\frac{\tau_{j}}{2 T}\right)\right) .
\end{aligned}
$$

The effect of the timing noise on the output error is estimated as significant if there exists a time instant $t_{j}$ such that $\varepsilon_{n t}\left(t_{j}\right)>\left(\varepsilon_{n u}\left(t_{j}\right)+\varepsilon_{n y}\left(t_{j}\right)\right) / 10$. For instance, if $u_{0}=$ $1, k=0.3, T=5, t_{j}=1, \tau_{j}=0.5, \sigma_{y}=0.01, \sigma_{u}=0.1^{1}$

\footnotetext{
${ }^{1}$ These values have been chosen from empirical knowledge of biologists and experimental results reported in [11].
}

then $\varepsilon_{n t}\left(t_{j}\right) \approx 5 \cdot 10^{-5}, \varepsilon_{n u}\left(t_{j}\right) \approx 3 \cdot 10^{-5}$ and $\varepsilon_{n y}\left(t_{j}\right) \approx$ $1 \cdot 10^{-4}$. Consequently, the effect of the timing noise on the output error cannot be neglected for the time instant $t_{j}=1(h)$. The impact of $n_{t}$ decreases as $t_{j}$ and becomes negligible from $t_{j} \gtrsim 3(h)$. This example emphasizes that $n_{t}$ could significantly influence the estimation of the time constant which mainly depends on the first measurement samples. Since the consequences of $n_{t}$ cannot be reasonably ignored, usual parameter estimation methods (those assuming only the presence of output noise) and error-in-variables approaches are not appropriate to solve this estimation problem. In the next section, a bounded-error parameter estimation approach is proposed as a suited solution to handle timing errors.

\section{BOUNDED-ERROR ESTIMATION WITH in vitro DATA}

Bounded-error or set-membership approaches allow to estimate parameters and their uncertainty in inverse problem contexts in which all uncertain quantities are assumed as unknown but bounded with known bounds. No further hypothesis about probability distributions is stated. Several algorithms have been developped to solve estimation problems stated in the bounded-error context. When models are non linear, most approaches use interval analysis and constraint propagation techniques. Allied with global algorithms and a reliable numerical implementation, they derive guaranteed computations, i.e. they provide a numerical proof of property or non-property. They are rather mature techniques and have already been successfully applied for solvling problems in biology, chemical or thermal engineering, economics, computer vision or robotics, when guaranteed computations were essential [8], [12].

In this part, we assume that all the uncertain quantities satisfy this bounded-error property and bounded-error estimation techniques are applied to experimental data collected during in vitro uptake kinetics experiments of a PS into human malignant glioma cells. The experimental protocol is defined by $N_{t}=5, N_{r}=6$ and $u_{0}=25 \mathrm{~mol}$.

TABLE II

PRIOR FEASIBLE INTERVALS FOR THE DATA

\begin{tabular}{cccc}
\hline$j$ & $t_{j}(\mathrm{~h})$ & {$\left[t_{j}\right]$} & {$\left[\hat{y}\left(t_{j}\right)\right]$} \\
\hline 1 & 1 & {$[0.67 ; 1.33]$} & {$[0 ; 0.607]$} \\
2 & 2 & {$[1.67 ; 2.33]$} & {$[0.238 ; 0.861]$} \\
3 & 8 & {$[7.67 ; 8.33]$} & {$[0.681 ; 1.396]$} \\
4 & 18 & {$[17.67 ; 18.33]$} & {$[0.661 ; 1.447]$} \\
5 & 24 & {$[23.67 ; 24.33]$} & {$[1.376 ; 2.459]$} \\
\hline
\end{tabular}

Fig. 4 presents the experimental data of six PS uptake kinetics carried out in the same experimental framework. Each cross corresponds to one measurement. The output variable measured by the spectrofluorimeter is given in arbitrary unit. Prior intervals $\left[\hat{y}\left(t_{j}\right)\right]$ and $\left[t_{j}\right]$ on the output measurements and the time instants are given in table II. Bounds of $\left[t_{j}\right]$ have been measured during the kinetics experiment. $\left[\hat{y}\left(t_{j}\right)\right]$ has been determined from the minimum and maximum values of measurements. The uncertainty associated with each pair of output and time data is materialized by a box. The set $\widehat{\mathbb{P}}$ 


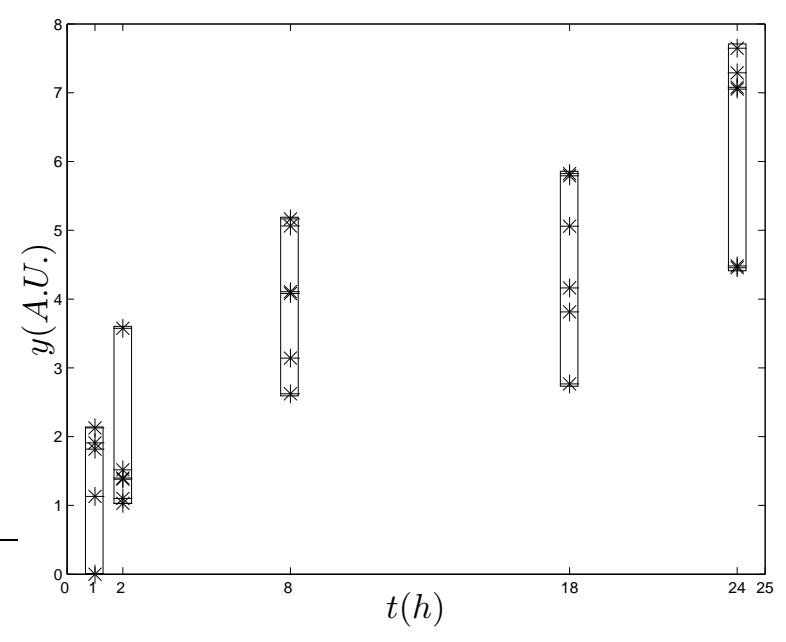

Fig. 4. Experimental data, intervals and boxes

to be characterized consists of all the values of $\mathbf{p}=(k, T)^{\prime}$ such that the graph of the function

$$
g(\mathbf{p}, t)=k \cdot u_{0} \cdot\left(1-e^{-t / T}\right)
$$

goes through all five boxes of Fig. 4. $\widehat{\mathbb{P}}$ is defined as

$$
\widehat{\mathbb{P}}=\left\{\mathbf{p} \in \mathbb{R}^{+2} \mid \exists \mathbf{t} \in \mathbb{R}^{+5},(g(\mathbf{p}, \mathbf{t}), \mathbf{p}, \mathbf{t}) \in \mathbb{X}\right\}
$$

with

$$
g(\mathbf{p}, \mathbf{t})=\left(g\left(\mathbf{p}, t_{1}\right) \quad \cdots \quad g\left(\mathbf{p}, t_{N_{t}}\right)\right)
$$

and

$$
\mathbb{X}=\left[\hat{y}\left(t_{1}\right)\right] \times \cdots \times\left[\hat{y}\left(t_{5}\right)\right] \times[\check{k}] \times[\check{T}] \times\left[t_{1}\right] \times \cdots \times\left[t_{5}\right],
$$

and the prior box for the parameters given by

$$
\check{\mathbb{P}}=[\check{k}] \times[\check{T}]=[1,4] \times[1,40] .
$$

$\widehat{\mathbb{P}}$ can be estimated in a guaranteed way using a set inversion algorithms based on parameter space partionning, interval analysis and constraint propagation techniques (see [8] and the references therein). Fig. 5 presents the estimate of $\widehat{\mathbb{P}}$ when the partionning algorithm is set not to partition boxes with a size smaller than 0.01

In Fig. 5, the paving form associated with $\widehat{\mathbb{P}}$ is composed of grey and black boxes. The grey boxes have been proved to be included in $\mathbb{P}$ but no conclusion has been reached for the black ones. The external envelope of $\widehat{\mathbb{P}}$ is defined by $\hat{k} \in[1.37 ; 3.49]$ and $\hat{T} \in[1.7 ; 33]$. This results shows that the estimation uncertainty on the time-constant is larger than the one on the static gain. Fig. 6 depicts the a posteriori estimate of the output set $\widehat{\mathbb{Y}}$, a set of time trajectories defined by

$$
\begin{array}{r}
\widehat{\mathbb{Y}}=\left\{(t, y) \in \mathbb{R}^{+} \times \mathbb{R} \mid y(t)=k u_{0}\left(1-e^{-t / T}\right),\right. \\
\text { with } \left.(k, T)^{T} \in \widehat{\mathbb{P}}\right\} .
\end{array}
$$

This figure points out a wide variation of the initial slope of the step response which explains the large uncertainty on the time-constant estimate. In this study case, the wide variation

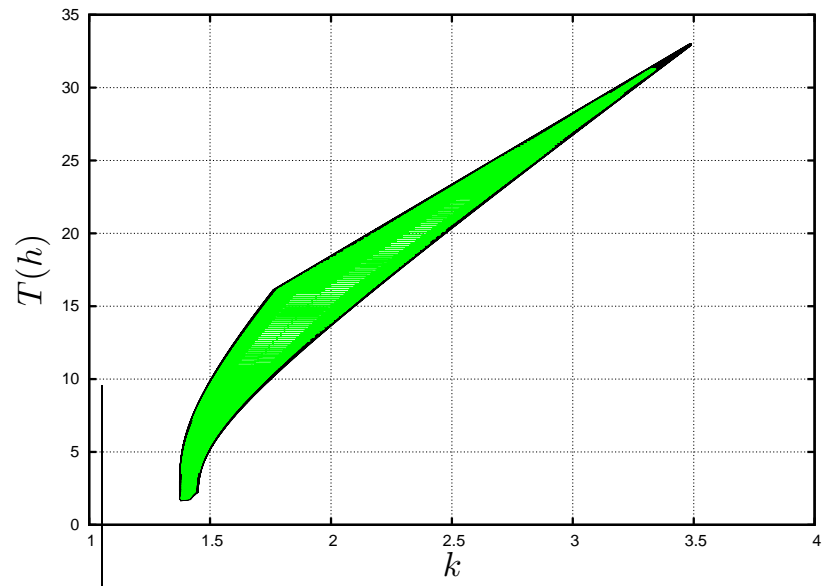

Fig. 5. a posteriori estimate of the parameter set $\widehat{\mathbb{P}}$

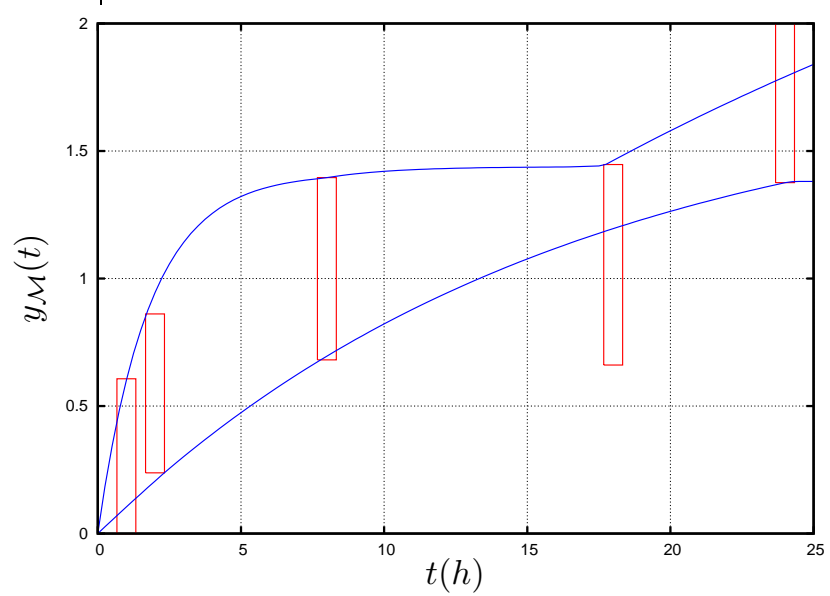

Fig. 6. a posteriori estimate of the output set $\widehat{\mathbb{Y}}$

of the initial slope is mainly due to the height of the boxes rather than their width. In other terms, in this application, the uncertainty on the time-constant estimate is mainly caused by the output noise rather than the timing noise.

\section{CONCLUSION}

This paper focuses on consequences of timing errors in collected data on the parameter estimation of kinetics models and more precisely their effects on the output error. The contribution of the timing noise on the output error is compared with the ones induced by input and output noise in terms of bias and variance. Mathematical expressions of the bias and variance of the output error with respect to the parameters of input, output and timing noises are established. It is shown that the influence of the timing noise on the output error can be significant, particularly for the first measurement time instants $\left(t_{j} \lesssim 3 h\right)$. An application to in vitro data is developed in the second part of this paper. It is shown how the timing noise can be taken into account by boundederror estimation algorithms based on interval analysis. The timing noise is described as a bounded error and no further hypothesis about probability distributions is stated. The results presented herein emphasize the effectiveness of such an 
bounded-error estimation approach in such an experimental framework.

\section{REFERENCES}

[1] M. Barberi-Heyob, P.-O. Védrine, J.-L. Merlin, R. Millon, J. Abecassis, M.-F. Poupon, and F. Guillemin, "Wild-type p53 gene transfer into mutated p53 HT29 cells improves sensitivity to photodynamic therapy via induction of apoptosis," Int. J. Oncol., no. 24, pp. 951-958, 2004.

[2] J. G. Moser, Photodynamic Tumor Therapy: 2nd and 3rd Generation. Gordon \& Breach Science Publishers, 1998.

[3] N. D. Evans, R. J. Errington, M. J. Chapman, P. J. Smith, M. J. Chappell, and K. R. Godfrey, "Compartmental modelling of the uptake kinetics of the anti-cancer agent topotecan in human breast cancer cells," International Journal of Adaptive Control and Signal Processing, vol. 19, pp. 395-417, 2005.

[4] C. L. Beck, H.-H. Lin, and M. Bloom, "Multivariable modeling and control of anesthetic pharmacodynamics," in Proc of the CNRSNSF Workshop on Biology and Control Theory: current challenges, Toulouse, France, April 2006.

[5] R. Ali, L. Campbell, N. D. Evans, R. J. Errington, K. R. Godfrey, P. J. Smith, and M. J. Chappell, "A PK-PD model of cell cycle response to topotecan," in Proc of the IFAC Modelling and Control in Biomedical Systems, Reims, France, September 2006, pp. 477-482.

[6] M. J. Niedre, A. J. Secord, M. S. Patterson, and B. C. Wilson, "In vivo tests of the validity of singlet oxygen luminescence measurements as a dose metric in photodynamic therapy," Cancer Research, vol. 63, pp. 7986-7994, november 2003.

[7] J. Kusuma and V. K. Goyal, "Signal parameter estimation in the presence of timing noise," in Proc of the 40th Conference on Information Science and Systems, CISS'06, Princeton, NJ, USA, March 2006.

[8] L. Jaulin, M. Kieffer, O. Didrit, and E. Walter, Applied Interval Analysis. Springer, 2001

[9] T. Bastogne, L. Tirand, M. Barberi-Heyob, and A. Richard, "Modélisation système de la thérapie photodynamique," in 4ème Conférence Internationale Francophone d'Automatique, Bordeaux, France, May-June 2006.

[10] E. Walter and L. Pronzato, Identification of Parametric Models from experimental data. Springer-Verlag, Masson, 1997.

[11] T. Bastogne, L. Tirand, M. Barberi-Heyob, and A. Richard, "System identification of photosensitiser uptake kinetics in photodynamic therapy," in Proc of the 6th IFAC Symposium on Modelling and Control in Biomedical System (including biological systems), Reims, France, Sept. 2006

[12] I. Braems, N. Ramdani, M. Kieffer, L. Jaulin, E. Walter, and Y. Candau, "Guaranteed characterization of thermal conductivity and diffusivity in presence of model uncertainty," accepted in Inverse Problems in Science and Engineering, 2006.

\section{APPENDIX I}

\section{Lemma 1.1:}

$$
\mathcal{E}\left\{e^{a\left(\frac{1}{2}-U\right)}\right\}=\frac{\sinh \left(\frac{a}{2}\right)}{\frac{a}{2}}=\operatorname{sinhc}\left(\frac{a}{2}\right),
$$

where $a$ is a non-null constant and $U$ is a random variable distributed according to a uniform law on $[0,1]$.

\section{APPENDIX II}

\section{PROOF OF THE PROPOSITION 5.1}

Proof: Since $G_{u}$ and $G_{y}^{j}$ are centered and since $U_{t}^{j}$ and $G_{u}$ are independent, then

$$
\begin{aligned}
\mathcal{E}\left\{e_{y}\left(t_{j}\right)\right\} & =k \cdot u_{0} \cdot\left(1-\mathcal{E}\left\{e^{-\frac{1}{T}\left(t_{j}-\frac{\tau_{j}}{2}+\tau_{j} U_{t}^{j}\right)}\right\}\right) \\
& -k \cdot u_{0} \cdot\left(1-e^{-\frac{t_{j}}{T}}\right) \\
& =k \cdot u_{0} \cdot e^{-\frac{t_{j}}{T}}\left(1-\mathcal{E}\left\{e^{-\frac{1}{T}\left(-\frac{\tau_{j}}{2}+\tau_{j} U_{t}^{j}\right)}\right\}\right) \\
& =k \cdot u_{0} \cdot e^{-\frac{t_{j}}{T}}\left(1-\mathcal{E}\left\{e^{\frac{\tau_{j}}{T}\left(\frac{1}{2}-U_{t}^{j}\right)}\right\}\right) .
\end{aligned}
$$

It can be deduced from Lemma 1.1 that

$$
\mathcal{E}\left\{e_{y}\left(t_{j}\right)\right\}=k \cdot u_{0} \cdot e^{-\frac{t_{j}}{T}}\left(1-\sinh c\left(\frac{\tau_{j}}{2 T}\right)\right) .
$$

\section{APPENDIX III}

ProOF OF THE PROPOSITION 5.2

Proof: From (16), $e_{y}\left(t_{j}\right)$ is rewritten such that

$$
e_{y}\left(t_{j}\right)=X_{1}+X_{2}-y_{\mathcal{M}}\left(t_{j}\right) \text {, }
$$

with

$$
\begin{aligned}
& X_{1}=k \cdot\left(u_{0}+\sigma_{u} G_{u}\right) \cdot\left(1-e^{-\frac{1}{T}\left(t_{j}-\frac{\tau_{j}}{2}+\tau_{j} U_{t}^{j}\right)}\right) \\
& X_{2}=\sigma_{y} G_{y}^{j} .
\end{aligned}
$$

Since $X_{1}$ and $X_{2}$ are independent, it can be deduced that

$$
\begin{aligned}
\operatorname{Var}\left(e_{y}\left(t_{j}\right)\right) & =\operatorname{Var}\left(X_{1}\right)+\operatorname{Var}\left(X_{2}\right) \\
& =\operatorname{Var}\left(X_{1}\right)+\sigma_{y}^{2} .
\end{aligned}
$$

Let us compute the expectation of $X_{1}$.

$$
\begin{aligned}
\mathcal{E}\left\{X_{1}\right\} & =k \cdot u_{0} \cdot\left(1-e^{\frac{-t_{j}}{T}} \mathcal{E}\left\{e^{\frac{\tau_{j}}{T}\left(\frac{1}{2}-U_{t}^{j}\right)}\right\}\right) \\
& =k \cdot u_{0} \cdot\left(1-e^{\frac{-t_{j}}{T}} \sinh c\left(\frac{\tau_{j}}{2 T}\right)\right),
\end{aligned}
$$

according to Lemma 1.1. The expectation of $X_{1}^{2}$ is given by

$$
\begin{aligned}
\mathcal{E}\left\{X_{1}^{2}\right\} & =k^{2} \mathcal{E}\left\{\left(u_{0}+\sigma_{u} G_{u}\right)^{2}\right\} \mathcal{E}\left\{X_{3}^{2}\right\} \\
& =k^{2}\left(u_{0}^{2}+\sigma_{u}^{2}\right) \mathcal{E}\left\{X_{3}^{2}\right\},
\end{aligned}
$$

where

$$
X_{3}=1-e^{-\frac{1}{T}\left(t_{j}-\frac{\tau_{j}}{2}+\tau_{j} U_{t}^{j}\right)} .
$$

We have

$$
\begin{aligned}
\mathcal{E}\left\{X_{3}^{2}\right\} & =1+\mathcal{E}\left\{e^{-\frac{2}{T}\left(t_{j}-\frac{\tau_{j}}{2}+\tau_{j} U_{t}^{j}\right)}\right\}-2 \mathcal{E}\left\{e^{-\frac{1}{T}\left(t_{j}-\frac{\tau_{j}}{2}+\tau_{j} U_{t}^{j}\right)}\right\} \\
& =1+e^{-\frac{2 t_{j}}{T}} \mathcal{E}\left\{e^{\frac{2 \tau_{j}}{T}\left(\frac{1}{2}-U_{t}^{j}\right)}\right\}-2 e^{-\frac{t_{j}}{T}} \mathcal{E}\left\{e^{\frac{\tau_{j}}{T}\left(\frac{1}{2}-U_{t}^{j}\right)}\right\} \\
& =1+e^{-\frac{2 t_{j}}{T}} \operatorname{sinhc}\left(\frac{\tau_{j}}{T}\right)-2 e^{-\frac{t_{j}}{T}} \sinh c\left(\frac{\tau_{j}}{2 T}\right) .
\end{aligned}
$$

We finally obtain

$$
\begin{aligned}
\operatorname{Var} & \left\{X_{1}\right\}=k^{2}\left(u_{0}^{2}+\sigma_{u}^{2}\right)\left(1+e^{-\frac{2 t_{j}}{T}} \sinh c\left(\frac{\tau_{j}}{T}\right)\right. \\
& \left.-2 e^{-\frac{t_{j}}{T}} \sinh c\left(\frac{\tau_{j}}{2 T}\right)\right)-k^{2} u_{0}^{2}\left(1-e^{-\frac{t_{j}}{T}} \sinh c\left(\frac{\tau_{j}}{2 T}\right)\right)^{2} \\
& =k^{2}\left(u_{0}^{2}+\sigma_{u}^{2}\right)\left(1+e^{-\frac{2 t_{j}}{T}} \sinh c\left(\frac{\tau_{j}}{T}\right)-2 e^{-\frac{t_{j}}{T}} \sinh c\left(\frac{\tau_{j}}{2 T}\right)\right) \\
& -k^{2} u_{0}^{2}\left(1-2 e^{-\frac{t_{j}}{T}} \sinh c\left(\frac{\tau_{j}}{2 T}\right)+e^{-\frac{2 t_{j}}{T}}\left(\sinh c\left(\frac{\tau_{j}}{2 T}\right)\right)^{2}\right) \\
& =k^{2}\left(\sigma_{u}^{2}+\left(u_{0}^{2}+\sigma_{u}^{2}\right) e^{-\frac{2 t_{j}}{T}} \sinh c\left(\frac{\tau_{j}}{T}\right)\right. \\
& \left.-2 \sigma_{u}^{2} e^{-\frac{t_{j}}{T}} \sinh c\left(\frac{\tau_{j}}{2 T}\right)-u_{0}^{2} e^{-\frac{2 t_{j}}{T}}\left(\sinh c\left(\frac{\tau_{j}}{2 T}\right)\right)^{2}\right) .
\end{aligned}
$$

Equation (18) in proposition 5.2 is then a direct consequence of (38). 